\title{
CRÓNICAS
}

Revista Atlántica-Mediterránea 22, pp. 436-439

BIBLID [2445-3072 (2020) 22, 1-443]

https://doi.org/10.25267/rev_atl-mediterr_prehist_arqueol_soc.2020.v22.33

\section{Ana BARROSO MUÑOZ}

Estudiante, Universidad de Cádiz.

Correo electrónico: anabarrosomu@gmail.com

Crónica del Seminario "NeoNet: Origen, desarrollo y consolidación del Neolítico en el ámbito Mediterráneo". Acciones de dinamización "Redes de Investigación" (RED2018-102382-T). Ministerio de Ciencia Innovación. Barcelona (online), 11-12 de noviembre de 2020.

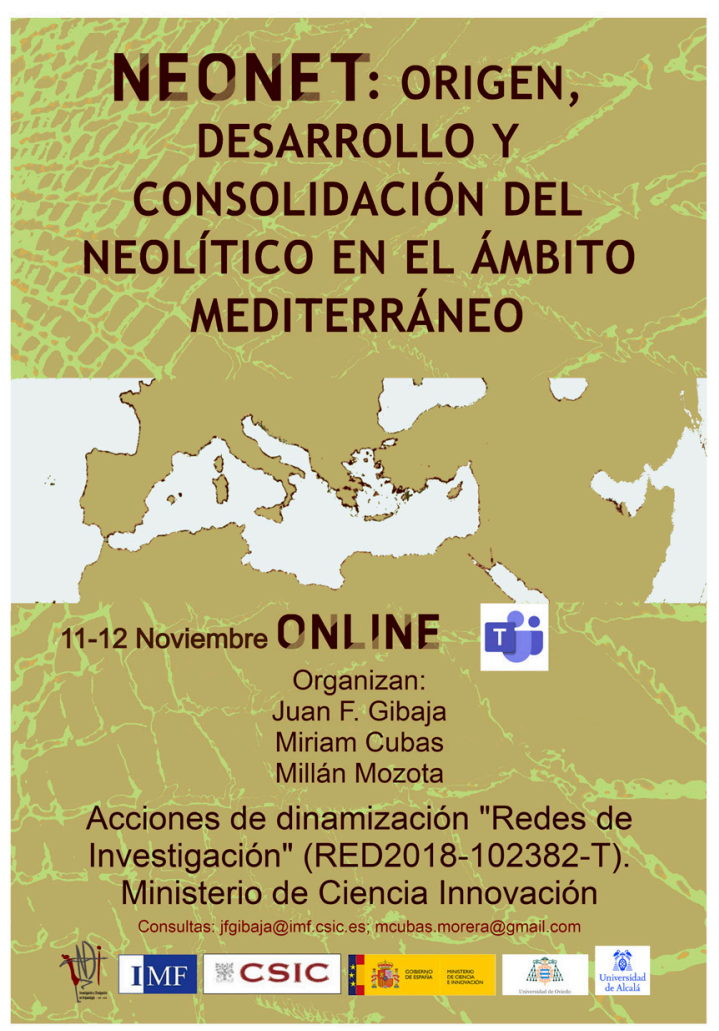

El desarrollo del seminario se produciría durante los días 11 y 12 de noviembre mediante la aplicación de videollamadas Microsoft Teams. Las sesiones serían online debido a la situación actual que no permite las reuniones y desplazamientos para llevar a cabo esta clase de proyectos. Este seminario forma parte del proyecto NeoNet, que tuvo su inicio en el año 2018 y del que ya se han celebrado un primer encuentro presencial en 2019 y la actual reunión online en 2020. El objetivo de dicho proyecto consiste en crear una red para el estudio del Neolítico en el sur de Europa con una vinculación de los investigadores a sus instituciones y laboratorios con la intención de que exista un intercambio de conocimientos científicos. Asimismo, se busca la movilidad y transferencia de los conocimientos para así poder formar a futuros investigadores. Por ello, el equipo directivo considera de gran importancia la divulgación de los trabajos y el impulso a nuevos proyectos nacionales e internacionales. Como diría el propio Juan Francisco Gibaja, uno de los directores del proyecto, lo más interesante para ellos es poder abrir puertas de colaboración entre los distintos investigadores y realizar así nuevas propuestas doctorales para ayudar a los jóvenes investigadores.

La recepción y las primeras sesiones del seminario se realizarían en el espacio de la mañana del día 11 de noviembre a las $9.00 \mathrm{~h}$ hasta las $13.30 \mathrm{~h}$. Esta recepción sería iniciada por Luis Calvo, director del Centro Mila y Fontanals del CSIC, quien resaltaría en su discurso la importancia de tener una red de conexión y comunicación continuada entre investigadores internacionales, la cual permitiría el trabajo en equipo para el desarrollo de futuras investigaciones sobre el Neolítico. A esta apertura le seguiría Juan Francisco Gibaja, de la Universidad Autónoma de Barcelona y científico en el Consejo Superior de Investigaciones Científicas (CSIC), quien se encargaría de explicar dicho proyecto y la estructuración de las jornadas. En esta ocasión, debido a su desarrollo virtual, ha podido llegar a un público más amplio, desde investigadores consolidados a estudiantes universitarios interesados en ampliar sus conocimientos.

Terminada la recepción y presentación del proyecto, iniciaría su ponencia Miriam Cubas, B. Comendador y A.F. Carvalho, centrada en el Neolítico en el Norte peninsular y Portugal. En concreto, explicarían los procesos de neolitización en todo el frente norte peninsular, incluyendo sus proyectos en Galicia, Asturias y el norte de Portugal, los cuales se enfocarían en la cerámica cardial y las pinturas esquemáticas.

Seguidamente, Íñigo García, M. A. Rojo y Cristina Tejedor pondrían en el punto de mira el Neolítico del Interior peninsular, concretamente en el área de la Meseta Central y el Valle Medio y Alto del Ebro. Para conocer cómo serían los inicios del Neolítico, sus objetos de estudios se centrarían tanto el registro arqueológico material como el ADN de restos óseos humanos. Con sus hallazgos, podrían establecer hasta dos fases de transformación hacia una economía plenamente productiva.

La siguiente ponencia estaría enfocada en el sur peninsular. De la mano de María Dolores Camalich y Dimas Martín, se presentarían los resultados de 
numerosos yacimientos tan importantes como la Cueva del Toro, la Cueva de Nerja o la Cueva de los Murciélagos. Estos dos investigadores habrían realizado estudio traceológicos en útiles líticos y estudio de residuos en cerámicas con el objetivo de aproximarse a la secuencia cronológica y evolutiva del Neolítico en Andalucía.

Tras una pausa de "café virtual", seguiría la ponencia de Joan Bernabeu y Oreto García, miembros del Grupo Premedoc, del Departamento de Prehistoria, Arqueología e Historia Antigua de la Universidad de Valencia. Su investigación se centra en el estudio del proceso de transición de las poblaciones mesolíticas a las neolíticas en el Levante peninsular, la cual situarían desde el 8500 al 4200 B.P.

Niccolò Mazzuco y Thomas Huet explicarían el Neolítico del Mediterráneo Central. El área de estudio desarrollada en esta ponencia se encontraría en el centro y sur de Europa, concretamente en el valle italiano, donde llevan a cabo proyectos en áreas con cronologías desde Mesolítico final al inicio del Neolítico.

A continuación, el doctor Juan José Ibáñez nos acercaría el Neolítico en el Próximo Oriente. Durante el desarrollo de su exposición, resaltaría las importantes transformaciones que se habrían producido con el inicio de la agricultura. Mediante estudios arqueobotánicos se habría podido analizar estos primeros intentos agrícolas en el sureste de Turquía.

La última ponencia del bloque de la mañana vendría de la mano de Eneko Iriarte, profesor en la Universidad de Burgos. Bajo el título "El clima durante el Neolítico", abarcaría todos los procesos tectónicos que tendrían lugar en el Holoceno y los cuales resultarían indispensables para comprender cómo sería el clima en el que vivieron las primeras sociedades humanas.

Por la tarde comenzaría el segundo bloque de ponencias virtuales. En este sentido, Marta Moreno, Patricia Martín y Guillem Pérez serían los encargados de tratar el tema de la domesticación animal y vegetal. Para ello, establecerían tres ejes fundamentales: la identificación taxonómica de los mamíferos, la distinción entre la cabaña doméstica y fauna salvaje y la aplicación de estudios isotópicos para conocer la alimentación de los animales.

La penúltima ponencia de la tarde versaría sobre el Neolítico del Nordeste peninsular, con los investigadores Xavier Oms y Javier Nadal ,quienes analizarían la situación actual de la investigación científica. En este sentido, harían una enérgica críti- ca a la ausencia de proyectos $\mathrm{y}$, consecuentemente, de documentación sobre el Mesolítico reciente en el área de la costa catalana.

La última ponencia de la tarde se realizaría en italiano por Mario Mineo sobre el asentamiento neolítico de la Marmotta. Este yacimiento subacuático excepcional habría sido descubierto casualmente en la construcción de un acueducto y resulta de gran interés para el estudio del Neolítico en diversos aspectos: innovación tecnológica, ocupación, evolución de los útiles y, especialmente, sobre la navegación, habiéndose documentado un total de 5 navíos. Situado en la costa tirrénica y próximo a Roma, se localiza en plena área del Neolítico cardial y es de especial interés para el conocimiento del lago como una importante vía de comunicación.

El tercer bloque de ponencias comenzaría el jueves por la mañana. Juan Francisco Gibaja, Niccolò Mazzuco, Alba Masclans y Millán Mozota analizarían las zonas de extracción de las materias primas, la morfología, las huellas de uso y las técnicas relacionadas con la microscopía confocal para el análisis de la lítica. Concluirían su ponencia con una crítica a la falta de estudios sobre la división sexual del trabajo, así como la necesidad de establecer una nueva línea de investigación basada en el análisis de los residuos.

A continuación, Miriam Cubas y Xavier Oms centrarían su estudio en la producción cerámica durante el Neolítico. La intervención comenzaría con un análisis del estado actual de la cuestión en el marco del suroeste peninsular. El estudio morfológico y el análisis estilístico de las decoraciones serían el eje conductor para el conocimiento somero de la cronología. No obstante, resaltarían la inexistencia de un sistema estándar para la descripción cerámica, con lo cual cada equipo elabora su propia metodología y esto repercute en la dificultad a la hora de llevar a cabo estudios comparativos.

Tras la última pausa del seminario, tendría lugar su clausura con la reunión de todos los miembros del proyecto. En breves minutos, expondrían los nuevos trabajos, propuestas y posibles líneas de investigación futuras para impulsar la colaboración internacional. Asimismo, elaborarían un balance positivo del seminario ante la enorme acogida que tendría por parte del público general interesado en esta temática. 